\title{
Acute neurological consequences of novel psychoactive substance use: a retrospective review in a large UK hospital
}

\author{
Authors: Matthew Tanti, ${ }^{A}$ Jeremy Cosgrove, ${ }^{B}$ Charles Kelleher, ${ }^{C}$ Rebekah Jones ${ }^{C}$ and Melissa Maguire ${ }^{B}$
}

\section{Background}

Novel psychoactive substances (NPS) are a growing public health concern. We aimed to identify the acute neurological consequences of NPS.

\section{Method}

We performed a retrospective case-note review of patients who presented to the emergency department after taking NPS.

Results

We identified 237 admissions from 190 patients, mostly young men. There were high rates of psychiatric comorbidity (43\%), unemployment $(39 \%)$, homelessness $(24 \%)$ and incarceration (17\%). Most reported use of synthetic cannabinoids (SC; $91 \%$ ). Some took synthetic cathinones (SCath; 7\%) or nitrous oxide (NOS; $2 \%$ ). SC caused impaired consciousness (61\%) and seizures (16\%). SCath users presented with psychiatric disturbance or seizures (55\%). Most patients were managed conservatively $(67 \%)$ and a small proportion (14\%) were referred to drug or psychology services.

\section{Conclusions}

NPS users represent a vulnerable group in society. Certain clinical features may suggest the type of NPS used. Most patients require supportive management and onward referral to drug addiction services is recommended.

KEYWORDS: novel psychoactive substances, synthetic cannabinoids, synthetic cathinones, nitrous oxide, neurology

DOI: $10.7861 /$ clinmed.2020-0706

\section{Introduction}

Novel psychoactive substances (NPS) are a public health concern due to their potency, addictive properties and ability to evade detection. ${ }^{1,2}$ They include synthetic cannabinoids (SC) like 'spice',

Authors: ${ }^{\text {A }}$ neurology registrar, Leeds Teaching Hospitals NHS Trust, Leeds, UK; ${ }^{B}$ consultant neurologist, Leeds Teaching Hospitals NHS Trust, Leeds, UK; ${ }^{C}$ medical student, Leeds School of Medicine, Leeds, UK synthetic cathinones (SCath) like 4-methyl methcathinone or 'MCAT', synthetic opioids, nitrous oxide (NOS) and new benzodiazepines. ${ }^{3}$ The European Monitoring Centre for Drugs and Drug Addiction recognises 620 different NPS: 169 of these are SC. ${ }^{4}$ Prior to the Psychoactive Substances Act in 2016, NPS were marketed as 'legal highs' in the UK; NPS suppliers can now face a 7 year prison sentence. ${ }^{5}$ NPS use is still prevalent. Emergency services in the north of England experienced a six-fold increase in NPS-related call-outs between 2015 and 2017. ${ }^{6}$ Furthermore, a UK-based drug survey reported an increase of NPS users from $17 \%$ to $27 \%$ between 2015 and 2017 . $^{7}$ The toxic effects of NPS are varied. Neurological consequences of NPS are described but no large case series exists to the best of our knowledge. ${ }^{8-10}$ This paper reports the acute neurological consequences of NPS in a large emergency department (ED) and examines risk factors associated with NPS use.

\section{Methods \\ Study design and inclusion criteria}

We performed a retrospective case-note review of patients attending the Leeds Teaching Hospitals NHS Trust ED. Electronic records between October 2016 and March 2019 were reviewed if they contained the following terms: 'NPS'; 'novel psychoactive substance'; 'legal high'; 'spice'; 'mephedrone'; 'MCAT'; 'meow meow'; 'laughing gas'; and 'nitrous oxide'. Records were included if NPS use was documented as the reason for admission. Records were excluded if the NPS type was unspecified, or if NPS use was unconfirmed.

\section{Data analysis}

Patient demographics and admission details were recorded. Vital signs and Glasgow Coma Scale score (GCS) were recorded as the first value documented by ED or ambulance crew. GCS was converted to ordinal data; severely impaired GCS was defined as 8 or less, moderately impaired as 9 to 12, and mildly impaired as 13 to 15. Percentages were presented as a proportion of the data available for the reported variable. Statistical testing was performed as an exploratory analysis with significance set at $p<0.05$. Chi-squared analysis was used for categorical data.

\section{Ethics approval}

This study was conducted in accordance with good clinical practice, data protection principles and local research and 
Table 1. Demographics of novel psychoactive substance users

\begin{tabular}{|c|c|c|c|c|c|c|}
\hline & $\begin{array}{l}\text { Total, } \\
n=190, \\
n(\%)\end{array}$ & $\begin{array}{l}\text { Synthetic } \\
\text { cannabinoid, } \\
n=146, n(\%)\end{array}$ & $\begin{array}{l}\text { Synthetic } \\
\text { cannabinoid } \\
\text { and additional } \\
\text { substance, } \\
n=23, n(\%)\end{array}$ & $\begin{array}{l}\text { Synthetic } \\
\text { cathinone, } \\
n=6, n(\%)\end{array}$ & $\begin{array}{l}\text { Synthetic } \\
\text { cathinone and } \\
\text { additional } \\
\text { substance, } \\
n=11, n(\%)\end{array}$ & $\begin{array}{l}\text { Nitrous } \\
\text { oxide, } \\
n=4, n(\%)\end{array}$ \\
\hline Male & $158(83)$ & $125(86)$ & $19(83)$ & $2(33)$ & $10(91)$ & $2(50)$ \\
\hline Age $\leq 34$ years & $108(59)$ & $81(56)$ & $10(48)$ & $5(84)$ & $8(72)$ & $4(100)$ \\
\hline Deceased & $15(8)$ & $11(8)$ & $4(17)$ & $0(0)$ & $0(0)$ & $0(0)$ \\
\hline \multicolumn{7}{|c|}{ Employment/housing status } \\
\hline Unemployed $^{a}$ & $57(39)$ & $43(36)$ & $11(58)$ & $1(25)$ & $2(67)$ & $0(0)$ \\
\hline Homeless & $35(24)$ & $30(25)$ & $5(26)$ & $0(0)$ & $0(0)$ & $0(0)$ \\
\hline Student & $15(10)$ & $9(8)$ & $1(5)$ & $3(75)$ & $1(33)$ & $1(50)$ \\
\hline \multicolumn{7}{|c|}{ Past medical history } \\
\hline Drug abuse & $82(53)$ & $62(51)$ & $15(75)$ & $1(25)$ & $4(57)$ & $0(0)$ \\
\hline Alcohol abuse & $41(26)$ & $35(29)$ & $6(30)$ & $0(0)$ & $0(0)$ & $0(0)$ \\
\hline Mental health & $67(43)$ & $51(42)$ & $11(55)$ & $1(25)$ & $4(57)$ & $0(0)$ \\
\hline Epilepsy/seizures & 23 (15) & 18 (15) & $3(15)$ & $0(0)$ & $2(29)$ & $0(0)$ \\
\hline
\end{tabular}

${ }^{a}$ Excluding homeless. Percentages are a function of available data, further breakdown in supplementary material S1.

development department guidance. To increase anonymity, age was categorised.

\section{Results}

We identified 332 admissions; 237 admissions from 190 patients met the inclusion/exclusion criteria (Tables 1 and 2). The responsible NPS agent was SC alone (SC-) in 188 (79\%) admissions, SC with additional substances (SC+) in $28(11 \%)$, SCath alone (SCath-) in six (2\%), SCath with additional substances $(\mathrm{SCath}+)$ in $11(5 \%)$, and NOS in four (1\%). No other NPS agents were reported. Supplementary tables are provided for additional data on demographics, presenting features, deceased patients and patients attending epilepsy clinic.

\section{Demographics}

Ages ranged from 13 to 60 years. Most were under 35 years old (59\%) and most were male ( $83 \%$; Table 1$)$. The majority were British ( $88 \%$ ) or single (97\%; supplementary material S1, Table S1). Socio-demographic data was obtained in 144 patients: 57 (39\%) were unemployed, an additional 35 (24\%) were homeless, 25 $(17 \%)$ were incarcerated and 15 were students $(10 \%)$.

Prior illicit substance use was identified in 49 (32\%) and a further $33(21 \%)$ reported intravenous drug use (supplementary material S1, Table S1). Alcoholism was frequently listed (26\%), as was prior seizures (15\%), liver cirrhosis (5\%) and viral hepatitis (14\%). Prior mental health issues were documented in 67 patients ( $43 \%$ ). Of these, 52 had a specified diagnosis, 26 (50\%) had depression, 11 (26\%) had anxiety, eight (15\%) had schizophrenia, six (12\%) had drug induced psychosis, five (10\%) had a personality disorder, three $(6 \%)$ had bipolar disorder, one $(2 \%)$ had post-traumatic stress disorder and four (10\%) had unspecified psychosis.

SC users were older than patients taking SCath or NOS: 73 in the SC groups ( $45 \%$ ) were aged 35 years or older compared with four $(19 \%)$ in the SCath and NOS groups $(n=185$; chi-squared $=4.95$; $p=0.026)$. SCath and NOS users were more likely to be students compared with SC users, five (56\%) compared with ten (7\%; $n=146$; chi-squared $=21.33 ; \mathrm{p}<0.001)$. No SCath or NOS users were homeless or incarcerated at the time of admission. Patient demographics between NPS subgroups were otherwise comparable.

\section{Synthetic cannabinoids without additional substances}

The most common reason for SC- admission was low GCS (Table 2). Low GCS was significantly associated with SC- use (61\%) compared with SC+, SCath and NOS combined (38\%; $n=231$; chi-squared $=8.27 ; p=0.004)$. Forty-two admissions $(26 \%)$ presented with severely impaired GCS (8 or less). Seizures were documented in 29 admissions (16\%); two patients (1\%) presented in status epilepticus. Psychiatric disturbance was documented in 16 admissions (9\%). Other transient neurological manifestations included two ( $1 \%)$ patients with tremor and one $(0.5 \%)$ with transient limb numbness. Some patients self-discharged (15\%), an additional $7 \%$ left before clinical assessment.

Investigations and management are summarised in supplementary material S1, Table S2. Of note, one patient had a positive urine toxicology screen for benzodiazepines. Raised creatine kinase ( $C K$; $>500 \mathrm{U} / \mathrm{L}$ ) was identified in five patients $(3 \%)$; two with a history of 'long lie', one with generalised seizure and two presented feeling 'generally unwell'. Computed tomography (CT) of the brain was performed in 12 patients. One patient with cardiac arrest had $\mathrm{CT}$ changes of hypoxic brain injury, but the rest were normal.

Most SC-admissions required observation only (72\%). Referral to drug services was made for 21 patients (12\%). A benzodiazepine was given to $11(6 \%)$ patients for seizures or psychiatric agitation. Only five patients were seen in neurology outpatients (supplementary material S1, Table S4). Most did not attend regular follow-up. Seizure frequency ranged from three per week to three 
Table 2. Admission details of novel psychoactive substance users

\begin{tabular}{|c|c|c|c|c|c|c|}
\hline & $\begin{array}{l}\text { Total, } \\
n=237 \\
n(\%)\end{array}$ & $\begin{array}{l}\text { Synthetic } \\
\text { cannabinoid, } \\
n=188, n(\%)\end{array}$ & $\begin{array}{l}\text { Synthetic } \\
\text { cannabinoid } \\
\text { and additional } \\
\text { substance, } \\
n=28, n(\%)\end{array}$ & $\begin{array}{l}\text { Synthetic } \\
\text { cathinone, } \\
n=6, n(\%)\end{array}$ & $\begin{array}{l}\text { Synthetic } \\
\text { cathinone and } \\
\text { additional } \\
\text { substance, } \\
n=11, n(\%)\end{array}$ & $\begin{array}{l}\text { Nitrous } \\
\text { oxide, } \\
n=4, n(\%)\end{array}$ \\
\hline Stayed for treatment & $142(80)$ & $121(81)$ & $13(72)$ & $2(67)$ & $3(75)$ & $3(100)$ \\
\hline Length of stay $<4$ hours & $122(60)$ & $106(63)$ & $13(54)$ & $1(33)$ & $1(25)$ & $1(50)$ \\
\hline \multicolumn{7}{|l|}{ Presenting symptom } \\
\hline Low GCS & $129(56)$ & $111(61)^{a}$ & $15(56)$ & $0(0)$ & $2(18)$ & $1(25)$ \\
\hline Seizure & $38(16)$ & $29(16)$ & $4(15)$ & $1(5)$ & $3(27)$ & $1(25)$ \\
\hline Psychiatric & $28(12)$ & $16(9)$ & $5(19)$ & $3(50)^{a}$ & $3(27)$ & $1(25)$ \\
\hline Headache & $5(2)$ & $5(3)$ & $0(0)$ & $0(0)$ & $0(0)$ & $0(0)$ \\
\hline Tremor & $3(1)$ & $2(1)$ & $0(0)$ & $1(5)$ & $0(0)$ & $0(0)$ \\
\hline Dystonia & $2(<1)$ & $0(0)$ & $1(4)$ & $0(0)$ & $1(9)$ & $0(0)$ \\
\hline Paraesthesia & $1(<1)$ & $1(<1)$ & $0(0)$ & $0(0)$ & $0(0)$ & $0(0)$ \\
\hline Abulia & $1(<1)$ & $0(0)$ & $0(0)$ & $1(5)$ & $0(0)$ & $0(0)$ \\
\hline Other & $24(10)$ & $19(10)$ & $2(7)$ & $0(0)$ & $2(18)$ & $1(25)$ \\
\hline \multicolumn{7}{|l|}{ Treatment } \\
\hline None & $140(66)$ & $125(72)$ & $8(32)$ & $1(25)$ & $4(67)$ & $2(100)$ \\
\hline Benzodiazepine & $13(6)$ & $11(6)$ & $2(8)$ & $0(0)$ & $0(0)$ & $0(0)$ \\
\hline Naloxone & $12(6)$ & $4(2)$ & $7(28)$ & $0(0)$ & $1(17)$ & $0(0)$ \\
\hline Counselling & $29(14)$ & $21(12)$ & $4(16)$ & $3(75)$ & $1(17)$ & $0(0)$ \\
\hline Other & $14(7)$ & $13(7)$ & $1(4)$ & $0(0)$ & $0(0)$ & $0(0)$ \\
\hline Procyclidine & $3(1)$ & $0(0)$ & $3(12)$ & $0(0)$ & $0(0)$ & $0(0)$ \\
\hline
\end{tabular}

${ }^{a}$ Chi-squared $p<0.005$ for presenting symptoms positively associated with NPS. GCS = Glasgow Coma Scale score. Percentages are a function of available data, further breakdown in supplementary material S1.

per year, and all except one patient with non-epileptic attacks received anti-epileptic medications.

\section{Synthetic cannabinoids and additional substances}

In total, there were 216 SC admissions but 28 of these took an additional substance at the time of admission (SC+). Prescribable medications were taken by 17 patients (such as morphine, pregabalin or antipsychotics) but in only three were they medically prescribed. Another five (18\%) took SC with cannabis and six (21\%) with heroin. Low GCS was responsible for 15 (54\%) admissions. $\mathrm{SC}+$ users were more likely to present with psychiatric disturbance than SC- users (18\% versus $9 \%$ ) but this difference was not statistically significant $(n=211$; chi-squared $=2.38 ; p=0.11)$.

Investigations and management are outlined in supplementary material S1, Table S2. One patient had a positive toxicology screen for morphine. Eight (35\%) SC+ patients were managed conservatively, significantly less than the $125(72 \%)$ in the SCgroup $(n=197$; chi-squared $=12.7 ; p<0.001$ ). Procyclidine was used for patients with suspected dystonic reactions in three cases of SC+ taken with heroin or antipsychotics. Naloxone was used in seven $(30 \%)$ cases, significantly more than the four $(2 \%)$ in the $S C$ - group $(n=197$; chi-squared $=21.6 ; p<0.001)$. A higher proportion of $\mathrm{SC}+$ admissions required a hospital stay over 24 hours compared with SC- (17\% versus 6\%) but the difference was not significant $(n=193$; chi-squared $=3.6 ; p=0.057$ ).

\section{Synthetic cathinones}

There were six admissions due to SCath- and 11 due to SCath+. Six (35\%) patients using SCath presented with psychiatric disturbance, a higher proportion than the 21 patients (10\%) using $S C(n=227$; chi-squared $=8.59 ; p=0.003)$. Psychiatric NPS were most likely due to SCath- compared with other NPS admissions combined $(50 \%$ versus $11 \% ; n=231$; chi-squared $=8.3 ; p=0.004$ )

No patients had a toxicology screen (supplementary material S1, Table S2). Two patients had a raised CK; one took SCath and methamphetamines leading to a CK of 3,900 U/L, dyskinesia, fever, trismus and muscle cramps. This patient received intravenous fluids but self-discharged within 4 hours. Many patients were simply observed ( $50 \%$ ), and four ( $40 \%$ ) were referred to drug services. Two $(28 \%)$ had a length of stay greater than a day.

\section{Nitrous oxide}

There were four admissions due to NOS; two used NOS with methylenedioxymethamphetamine (MDMA) and cocaine, one presented with psychosis and the other with seizure. All were conservatively managed (supplementary material S1, Table S2).

\section{Additional outcomes}

Overall, most patients were conservatively managed (67\%) and $122(60 \%)$ left the hospital within 4 hours (supplementary 
material S1, Table S2). Accidental exposure to SC- was reported in seven (3\%) admissions; all were discharged within 4 hours after monitoring. Four of the seven were prison workers.

Our cohort included 15 (8\%) deceased patients (supplementary material S1, Table S3). A cause of death was available in the medical records for five patients (33\%); two died from liver failure, one due to suicide, one from heroin overdose and one death directly related to NPS, the latter was a result of SC- with cardiac arrest and hypoxic brain injury. All 15 deceased patients used SC. All were male. Patients in the deceased cohort were more likely to be aged 35 or older ( $13 \%$ versus $5 \%$; $n=185$; chi-squared $=4.21$; $p=0.04)$. They were also more likely to have a history of intravenous drug use ( $40 \%$ versus $17 \% ; n=170$; chi-squared $=4.83 ; p=0.03)$, alcohol abuse $(73 \%$ versus $19 \%$; $n=170$; chi-squared $=21.77$; $p<0.001)$, liver cirrhosis ( $27 \%$ versus $3 \%$; $n=170$; chi-squared $=$ $17.69 ; p<0.001)$ and be unemployed $(100 \%$ versus $60 \%$; $n=151$; chi-squared $=9.27 ; p=0.002$ ).

\section{Discussion}

The UK lifetime prevalence of NPS use is estimated to be $2.5 \%$ in the Office for National Statistics (ONS) crime survey 2017-18. ${ }^{5}$ The actual figure may be higher as the ONS relied on household surveys and NPS users tend to be unemployed, homeless, students or prisoners. ${ }^{3,11}$ Young men are four times more likely than the general population to use NPS and this is reflected in our study demographics. ${ }^{5}$ In our cohort, $43 \%$ of patients had a prior mental health diagnosis. An association between NPS use and mental health is clear. In a review of 388 mental health admissions $50(13 \%)$ were due to NPS and 85 patients (22\%) were NPS users. ${ }^{12}$ Mental health issues and NPS use could have bidirectional associations.

SC or 'spice' was responsible for $90 \%$ of NPS admissions in our cohort and 11\% of those took SC and an additional agent. The 2016 Global Drugs Survey (GDS) ranked SC first in substances leading to ED visitation proportionate to use (inclusive of alcohol, tobacco and caffeine tablets). ${ }^{13}$ Of note, SC users had a 30 -fold increased ED visit risk than cannabis users. ${ }^{13}$ More recent findings suggest that harmful SC use might be reducing; the 2019 GDS ranked SC third in ED visit risk, behind methamphetamine and heroin. ${ }^{1}$ In our cohort, SC admissions were mainly due to reduced GCS (60\%) and $26 \%$ had a severely reduced GCS ( 8 or less). Seizures (16\%) and psychiatric manifestations (10\%) were also prominent. Dystonic reactions occurred in $1 \%$ of SC cases, but all occurred with co-administration of heroin or an antipsychotic. Previous case reports of neurological sequalae of SC include reversible cerebral vasoconstriction syndrome, new onset refractory status epilepticus and possible cardioembolic ischaemic stroke. ${ }^{8-10,14}$

The neurological effects of SC are likely a result of downstream neurotransmitter inhibition, but the mechanisms underlying impaired consciousness, seizures and psychiatric disturbances are unclear and may vary between different SC products. ${ }^{15}$ SC have a functional group structurally similar to delta-9tetrahydrocannabinol (THC), the psychoactive component of cannabis, but SC do not contain cannabidiol (CBD) which has antipsychotic and anxiolytic effects. ${ }^{16,17}$ THC and CBD act on endocannabinoid receptors. These are widely distributed in mammalian brains with high numbers in the basal ganglia, hippocampus, cerebral cortex and cerebellum. ${ }^{15,18}$ Compared with cannabis, $\mathrm{SC}$ binds with a higher affinity to $\mathrm{THC}$ receptors resulting in increased potency, addiction, adverse effects and withdrawal symptoms. ${ }^{15,19}$ Dosing with SC is also unpredictable. This is an issue with unregulated drug products, supporting arguments to legalise and regulate recreational drug use. ${ }^{20}$

SCath are stimulants like the cathinones found in khat, a highaltitude shrub grown in east Africa and the Arabian peninsula. ${ }^{21}$ Their effects and chemical structure are similar to amphetamines, MDMA and cocaine. Stimulant drugs increase synaptic levels of dopamine, serotonin and noradrenaline. ${ }^{19}$ SCath share the dopamine releasing properties of amphetamine and serotonin releasing properties of MDMA. ${ }^{22}$ There were 17 SCath ED admissions in our cohort and $35 \%$ of patients presented with psychiatric disturbance. Seizures occurred in $25 \%$, and $18 \%$ had transient neurological symptoms like dystonia, tremor or muscle weakness. In the UK, SCath was once as prevalent as conventional illicit drugs but popularity has declined in the past 5 years. $^{23}$

The 2019 GDS found NOS to be the 13th most popular recreational substance. ${ }^{1}$ Only four patients in our study attended ED following its use, either due to seizure, low GCS or psychiatric disturbance. NOS is a volatile substance with uncertain mode of action, it has short-lasting dissociative and euphoric effects. ${ }^{24}$ SCath and NOS users were mainly students, unlike SC users. Registered deaths due to NPS are rare but increasing; the ONS linked NPS to 750 deaths between 2004 and 2018, with 505 deaths since $2014 .{ }^{25}$ Our study cohort had a high mortality rate for their age: $15(8 \%)$ deceased at the time of data collection. Concurrent drug use, alcoholism and liver failure were more likely in this group and there was one documented suicide. We identified one death directly due to NPS in our study: a patient with hypoxic brain injury following SC use.

\section{Recommendations for clinicians}

Useful recommendations for the management of NPS patients are available from poisons services like TOXBASE, but the specific NPS agent needs to be identified. Unfortunately, drug screens do not detect NPS substances but they are still valuable as concurrent substance ingestion was high in our cohort $(21 \%)$ and a reversible compound might be identified. Commercial detecting kits are available for some common NPS, but most substances are only identified by specialised techniques like gas chromatography. ${ }^{26}$ Liaison with a local laboratory or poisons services would be needed. Only two patients had a drug screen in our study (supplementary material S1, Table S2). This is due, in part, to selection bias.

Presenting features are insufficient in determining NPS substance use, but patients presenting with low GCS were significantly more likely to have taken SC alone compared with other NPS in our study (61\% versus $38 \%$ ) and patients presenting with psychiatric disturbance were significantly more likely to have taken SCath alone compared with other NPS (50\% versus $11 \%$ ).

Acute NPS treatment is aimed at controlling complications such as airway compromise, seizures, psychosis, arrythmias and rhabdomyolysis. In our cohort, most patients (66\%) simply required monitoring and were either discharged or able to selfdischarge the same day (92\%). A referral to drug services was offered in only $14 \%$ of admissions in this study, a low number potentially attenuated by the high self-discharge rate of $20 \%$ (supplementary material S1, Table S2). Recovery in problem drug users is challenging but access to drug services is associated with significant improvements and should be encouraged at healthcare encounters. ${ }^{27}$ 


\section{Limitations}

There are several limitations to this retrospective case note review. Firstly, patients could not be included if they failed to report NPS use. Secondly, data collection relied on identifying key terms in the ED electronic records. We cannot determine how accurate this method was. For these reasons, it is likely that our data is an underestimate of NPS admissions to our ED department.

It is difficult to confidently attribute NPS outcomes to a specific NPS without a reliable testing method. Patients or clinicians may not have known which NPS was taken. There could be unreported concurrent drug use or accidental ingestion of other substances due to impure or contaminated products.

The long-term health consequences of NPS use are uncertain. By focusing on acute attendances, we could only make limited associations between NPS users and various comorbidities or socioeconomic outcomes, and limited observations on patients attending seizure clinics.

\section{Conclusion}

We identified 237 ED admissions due to NPS over a 29-month period. SC (often referred to as 'spice') resulted in most admissions due to reduced GCS, seizures and psychiatric manifestations. The medical management of these patients is largely supportive. SC users represent a vulnerable subgroup in society with high levels of additional substance abuse, mental health issues, unemployment, homelessness and incarceration. One patient died as a direct result of using SC in our cohort. Clinicians should be aware that NPS, particularly SC, are potent, highly addictive, evade detection by conventional drug testing methods and have toxic central nervous system effects.

\section{Summary}

\section{What is known?}

There are a growing number of novel psychoactive substances (NPS). They are potent drugs that evade detection by conventional hospital methods and have varied clinical effects.

\section{What is the question?}

What are the neurological complications of NPS use in the acute setting?

\section{What was found?}

NPS users were likely to be young men. There were high levels of prior substance misuse (53\%), mental health issues ( $43 \%$ ), unemployment (39\%), homelessness (24\%) and known epilepsy $(15 \%)$. Recurrent emergency department attendance due to NPS use was high ( $26 \%$ ). Synthetic cannabinoids (often known as 'spice') were responsible for most admissions, leading to low consciousness levels $(61 \%)$ or seizures $(16 \%)$. Patients taking synthetic cathinones were likely to present with psychiatric manifestations (35\%). Treatment of NPS use was mostly supportive $(67 \%)$.

\section{What is the implication for practice now?}

NPS should be suspected in young men presenting to ED with unexplained reduced consciousness, psychiatric disturbance or seizures, particularly if there is history of prior substance misuse. Acute treatment for NPS is largely supportive. Given high re-attendance rates and associated substance misuse in NPS users, referral for drug counselling should be considered in all cases.

\section{Supplementary material}

Additional supplementary material may be found in the online version of this article at www.rcpjournals.org/clinmedicine: S1 - Supplementary tables.

\section{Acknowledgements}

The authors would like to thank the data analysis team, particularly Sheona Gillies for identifying records.

\section{References}

1 Winstock A. Global Drug Survey (GDS) Key findings report: executive summary. London: Global Drug Survey, 2019. www.globaldrug survey.com/gds-2019 [Accessed 17 August 2020].

2 King L, Kicman A. A brief history of 'new psychoactive substances.' Drug Test Anal 2011;3:401-3.

3 The European Monitoring Centre for Drugs and Drug Addiction. New psychoactive substances in prison. EMCDDA, 2018. www. emcdda.europa.eu/countries/drug-reports/2018/united-kingdom/ drug-use_en [Accessed 17 August 2020].

4 The European Monitoring Centre for Drugs and Drug Addiction. Synthetic cannabinoids in Europe. EMCDDA, 2015. www.emcdda. europa.eu/topics/pods/synthetic-cannabinoids [Accessed 17 August 2020].

5 Flatley J. Drug Misuse: Findings from the 2017/18 Crime Survey for England and Wales. London: Office for National Statistics, 2018. www.gov.uk/government/statistics/drug-misuse-findings-from-the2017-to-2018-csew [Accessed 17 August 2020].

6 Marsh S. Police in Lancashire tackle surge of spice-related incidents. London: The Guardian, 2017. www.theguardian.com/society/2017/ apr/27/police-in-lancashire-tackle-surge-of-spice-related-incidents [Accessed 17 August 2020].

7 Deligianni E, Daniel OJ, Corkery JM, Schifano F, Lione LA: Impact of the UK Psychoactive Substances Act on awareness, use, experiences and knowledge of potential associated health risks of novel psychoactive substances. Br J Clin Pharmacol 2020;86:505-16.

8 Babi M-A, Robinson CP, Maciel CB. A spicy status: Synthetic cannabinoid (spice) use and new-onset refractory status epilepticusA case report and review of the literature. SAGE Open Med Case Reports 2017:5:2050313X1774520.

9 Freeman MJ, Rose DZ, Myers MA, et al. Ischemic stroke after use of the synthetic marijuana "spice." Neurology 2013;81:2090-3.

10 Stack C. A case of spice use with subsequent withdrawal and posterior reversible encephalopathy syndrome (PRES). Neurology 2017:88:P5.046.

11 Dargan PI, Albert S, Wood DM. Mephedrone use and associated adverse effects in school and college/university students before the UK legislation change. QJM 2010;103:875-9.

12 Stanley JL, Mogford DV, Lawrence RJ, Lawrie SM. Use of novel psychoactive substances by inpatients on general adult psychiatric wards. BMJ Open 2016:6:1-7.

13 Winstock A. Global Drug Survey (GDS) 2015 findings. London: Global Drug Survey, 2015. www.globaldrugsurvey.com/the-globaldrug-survey-2015-findings [Accessed 17 August 2020].

14 Heath TS, Burroughs Z, Thompson AJ, Tecklenburg FW. Acute intoxication caused by a synthetic cannabinoid in two adolescents. J Pediatr Pharmacol Ther 2012;17:177-81.

15 Basavarajappa BS, Subbanna S. Potential mechanisms underlying the deleterious effects of synthetic cannabinoids found in spice/K2 products. Brain Sci 2019;9:14.

16 The European Monitoring Centre for Drugs and Drug Addiction. Understanding the 'Spice' phenomenon. EMCDDA, 2009. www. emcdda.europa.eu/publications/thematic-papers/understandingspice-phenomenon_en [Accessed 17 August 2020]. 
17 Cohen K, Weinstein A. The effects of cannabinoids on executive functions: Evidence from cannabis and synthetic cannabinoids-a systematic review. Brain Sci 2018;8:40.

18 Thomas BF. Interactions of cannabinoids with biochemical substrates. Subst Abus Res Treat 2017;11:1-9.

19 Tracy DK, Wood DM, Baumeister D. Novel psychoactive substances: Types, mechanisms of action, and effects. BMJ 2017;356:1-8.

20 Godlee F. Drugs should be legalised, regulated, and taxed. BM] 2018;361:2057.

21 The European Monitoring Centre for Drugs and Drug Addiction. Khat drug profile. EMCDDA, 2015. www.emcdda.europa.eu/ publications/drug-profiles/khat [Accessed 17 August 2020].

22 Simmler LD, Buser TA, Donzelli M et al. Pharmacological characterization of designer cathinones in vitro. $\mathrm{Br}$ J Pharmacol 2013;168:458-70.

23 The European Monitoring Centre for Drugs and Drug Addiction. United Kingdom Country Drug Report. EMCDDA, 2018. www. emcdda.europa.eu/countries/drug-reports/2018/united-kingdom/ drug-use_en [Accessed 17 August 2020].

24 Randhawa G, Bodenham $A$. The increasing recreational use of nitrous oxide: History revisited. $\mathrm{Br}$ ] Anaesth 2016;116:321-4.
25 John E. Deaths related to drug poisoning by selected substances. Newport: Office for National Statistics (ONS), 2018. www.ons.gov. uk/peoplepopulationandcommunity/birthsdeathsandmarriages/ deaths/datasets/deathsrelatedtodrugpoisoningbyselectedsubstances [Accessed 17 August 2020].

26 Dei Cas M, Casagni E, Arnoldi S, Gambaro V, Roda G. Screening of new psychoactive substances (NPS) by gas-chromatography/time of flight mass spectrometry (GC/MS-TOF) and application to 63 cases of judicial seizure. Forensic Sci Int Synerg 2019;1:71-8.

27 Best D, Rome A, Hanning KA et al. Research for Recovery: A Review of the Drugs Evidence Base. Edinburgh: Scottish Government Social Research, 2010. www2.gov.scot/resource/doc/321958/0103435. pdf [Accessed 17 August 2020].

Address for correspondence: Dr Matthew Tanti, Department of Neurology, Leeds General Infirmary, Great George Street, Leeds LS1 3EX, UK.

Email:m.tanti@nhs.net

Twitter: @Matt_Tanti 